for many weeks. This contrast is very similar to that between myxomatosis and yellow fever.

Finally, the damage which virus infection inflicts on the host was discussed by Dr. J. C. N. Westwood. (Miorobiological Research Establishment, Porton) in relation to the intact host and by Dr. Alison Newton (University of Cambridge) at the cellular level. Dr. Westwood discussed "Virus Toxicity" and its possible role in the production of disease and ultimately death in fatal virus infections. The term 'virus toxicity' was originally coined to cover the production, by massive doses of virus, of lesions or death in susceptjble or non-susceptible animals without the occurrence of multiplication of the virus. The toxic factor has always been inseparably associated with the virus particle itself. Since virus disease is normally initiated by small doses of virus and accompanied by multiplication, it has seemed unlikely that 'toxicity' could be involved; and the latter has always been regarded as a freak laboratory phenomenon unassociated with production of disease. It was suggested that this concept might be wrong. Experiments were described which showed that the toxicity of rabbit pox virus is associated with a partial cycle of replication, as is the toxicity of other viruses in which such partial cycles have been sought. Death in rabbit pox is preceded by the abrupt onset of shock, with some evidence of increased capillary permeability, and the clinical picture can best be explained by the presence of some toxic factor in the blood. During the four days preceding death in acutely fatal infections, the virus concentration in the blood may rise to as high as $10^{6}$ infective units $/ \mathrm{ml}$. and similar. concentrations oceur in fatal smallpox and herpes infections in man. The cytotoxic effect of such virus concentrations on the capillary endothelium could be the cause of the increase in capillary permeability and so precipitate the animal into shock. Since no toxic factor other than the virus has ever been demonstrated to result from cell-virus interaction, the possible role of direct virus toxicity in disease must be seriously considered.

Damage to the intact host depends on the damage produced at the cellular level, and Dr. Newton considered the "Biochemical Aspects of Virus Infectivity" in relation to modification of cellular metabolism. The main modifications are found first in the production of new virus and second in changes in the nucleic acid and protein synthesis. Viruses which multiply in the cytoplasm make less use of the cell metabolism-for example, vaccinia virus does not affect the metabolism of deoxyribonucleic acid-and such viruses probably produce their damaging effects by competition for metabolites. Those which multiply in the nucleus produce a more profound effect, since they must not only compete for metabolites but must also overcome the normal control processes of the cell. Thus pre-labelling of the cellular deoxyribonucleic acid with tracer thymidine shows that herpes virus causes a rapid breakdown of cell deoxyribonucleic acid probably due to increased deoxyribonuclease activity similar to the increased ribonuclease activity stimulated by poliovirus. It also stimulates synthesis of deoxyribonucleic acid. These changes must damage the control mechanisms of the cell, and interfere with synthetic activities in the host. In some viruses, such as encephalomyocarditis, viral ribonucleic acid is elaborated in the nucleus and protein in the cytoplasm. It is probable that in these instances cytoplasmic protein metabolism is governed by viral ribonucleic acid which migrates from the nucleus. The production of cell damage does not depend on completion of the multiplication cycle of the virus; for example, with poliovirus, herpes and rabbit pox viruses, inhibition of infective virus production does not prevent cell destruction. It was suggested that cell damage results from the release of lysozomos, disruption of the controlling mechanism of the cell, competition for metabolites and disturbance of metabolic balance.

The meeting was successful in provoking lively discussion and considerable disagreement on nearly every point raised by the main speakers. Dr. Sanders, in summing up, congratulated the participants on avoiding all reference to infective nucleic acids, and he also stressed the gulf which still exists between academic virology and virology as applied to disease. J. C. N. Westwood

\title{
COTTON-GROWING IN NIGERIA
}

A GRICULTURAL progress relies on the pure sciences, on engineering and on economics. These cannot come into play until major practical problems have been resolved into their scientific components. Resolution is one of the functions of husbandry investigation. Recognition of the nature and importance of this function has come only within the past three decades. In this same period the other function of husbandry investigation has also been developed, namely, improvement of accepted practices in raising crops and livestock. Cultivations, drainage, sowing, grazing, housing of stock and other day-to-day matters were not looked on, formerly, as problems amenable to study : they were matters for the farmer's experience and tradition. Modern husbandry investigation seeks to improve all working practices by studying their underlying purposes and interpreting their effects.

That physical environment is primarily important for agriculture is now an axiom, though some costly flouting of it is yet fresh in memory. It is largely through husbandry investigation that scientific attention has been brought to its present intensity in such matters as rainfall characteristics, solar radiation, air temperature, shade, wind and moistureholding capacity of soils.

Some highly successful husbandry investigations on cotton have recently been reported* from the Ministry of Agriculture Research Station at Samaru, Northern Nigeria.

Past work at the Station and in its area of interest had revealed a discouraging situation. Local yields were 150-200 lb. of seed cotton an acre. Late sowing partly accounted for this, but earlier sowing in experimental plots gave only $240 \mathrm{lb}$. Assisted by fertilizers and by early planting the maximum yield was $600 \mathrm{lb}$. At these yields insecticidal protection did not pay. Yield in a bad year was only half of that in a good one and in any small field good and bad patches markedly influenced growth and yield. An improved variety of cotton, bred at the Station,

* Lawes, D. A., Emp. J. Exp. Agric., 29, 116 (1961). 
showed but a poor increase in yield (its merits were made clear in later trials under improved conditions). A belief had therefore grown up that the attainable yield of rain-grown cotton in the area was decidedly lower than that for other African territories.

Rainfall (total and effective) appeared to be the dominating factor for yield, and experiments were made during 1957-59 to assess its influence. From meteorological records and observations, 40 in. was deduced as the expected ( $1: 1$ chance) annual rainfall, $27.4 \mathrm{in}$. of which would come after July 1 ; and under local conditions a crop of cotton would need about 25 in. of rain in the growing season if growth was not to be limited by soil moisture (July 1 is recommended as the sowing dato for cotton, as the earliest which allows food crops to be planted first). What happens to rain after reaching the soil and how control of this influences yield were the primary issues in the field experiments.

Surface 'capping' through heavy rain, to which the local soils are specially prone, was shown to be largely controllable by timely hoeing and by mulching, entry of rain into the soil being thus greatly increased. The land is ridged for cotton and other crops, and it was found that here, as elsewhere, water: conservation could be improved by 'tying' the ridges with small bunds at intervals along the furrows. In dry years this considerably increased yield but depressed it in wet years. These effects were clearly interpreted by soil-moisture profiles.

The effects of varying degrees of these controls hav ing thus boen measured, field trials were arranged, having in mind steps which could be fitted into peasant practice. A light mulching, in the furrow bottoms only, proved effective, and bv slitting the furrow bottom and working a small amount of mulch into the slit the effect was enbanced. Tying alternate ridges only worked well, and avoided yield depression through standing water in wet years. Groundnut shell, dead grass and chopped Sorghum stalks made good mulches, the shell being best. By preparing the land for sowing at the onset of the rains, instead of just before sowing, which is local practice, cotton yields were raised by 15 to 20 per cent in two trials. Increased penetration and retention of rain had evidently been secured. Good response to fertilizers was obtained where tied ridges and mulch were also used.

The practical inference was clear-fertilizers, insecticidal protection, improved varieties and other betterments are repaid only when water conservation is amply ensured and this is practicable at peasant husbandry level. To drive the lesson home, cotton was grown on a half-acre plot for four years running in which rainfall was $55,35,42$ and 43 in. Yields were all of the order of $2,000 \mathrm{lb}$. of seed cotton an acre, which is good for rain-grown cotton anywhere and excellent in comparison with any other part of Africa. Moreover, picking was ended by Christmas whereas it dawdled on for another 2-3 months on other plots.

This successful husbandry investigation is of more than local and immediate value. It is part of the widespread studies of rainfall, soil moisture and crop water needs carried out by the Empire Cotton Grow. ing Corporation. The basic work is done at its Central Research Station, Namulonge, Uganda. All this work deals not with cotton alone but also in conjunction with the customary food crops of cotton growers. Agriculture in general is thus benefited in cotton-producing countries. Moreover, the practical problems centring on rainfall are becoming so defined and resolved as to enable their deeper, physiological study to be effectively opened up. Plant breeding, also, gains a clearer sense of direction.

\section{F. L. Engledow}

\section{RADIOMETRIC AND PHOTOMETRIC STANDARDS}

$\mathrm{T}$ WO recent publications in the series Notes on Applied Science of the National Physical Laboratory, Teddington, illustrate some of the activities of the Light Division of the Laboratory.

No. 23 in the series ${ }^{1}$, Radiometric Standards and Measurements, by E. J. Gillham, deals with the methods used at the Laboratory for the measurement of ultra-violet, visible and infra-red radiation. The contents consist of three main sections devoted to the measurement of total radiation, the measurement of monochromatic radiation, and the absolute measurement of radiation, respectively. Total radiation is usually more intense than monochromatic radiation and therefore can be measured more easily, but the total energy content of a bearn of radiation is often of less practical significance. An instrument for measuring total radiation must be equally sensitive to radiation at all wave-lengths, and the only means of converting radiation into another form of energy with predictable efficiency ovor the whole wavelength range is by absorption at a blackened surface, that is, to use a thermal dotector. Fortunately, blacks with an absorption factor of 95 per cent or more over the whole spectral range of interest are available, and consequently thermal detectors with an error due to selectivity of not more than 2 or 3 per cent can be obtained. The type of thermal detector commonly used is the thermopile, and in the booklet the Moll thermopile, which has steble characteristics and which forms a very useful working standard for measuring radiation or for calibration of other radiation detectors, is described in some detail. A set of six Moll thermopiles is maintained at tho National Physical Laboratory for calibration purposes. The methods used for the intercomparison of thermopiles and for the accurate measurement of the selectivity of a thermopile using a Callender radiobalance and three different sources of radiation are discussed.

To illustrate the problems encountered in the measurement of monochromatic radiation a description is given of a routine test undertaken at the Laboratory to determine the special sensitivity of a photoemissive cell, in the $0 \cdot 25-0 \cdot 7 \mu$ wave-length range. The optical arrangements and apparatus required for the measurement of the spectral sensitivity, and also the absolute sensitivity of the photocell, are given. For the absolute measurement of radiation a thermal detector in which the target is provided with a resistance element or other means for supplying a measurable amount of electrical power is recommended. At the National Physical Laboratory the absolute radiometer used is the Guild drift radiometer. It is of the compensated type with 